\title{
Quantifying the relative roles of selective and neutral processes in defining eukaryotic microbial communities
}

\author{
Peter Morrison-Whittle and Matthew R Goddard \\ The School of Biological Sciences, The University of Auckland, Auckland, New Zealand
}

\begin{abstract}
We have a limited understanding of the relative contributions of different processes that regulate microbial communities, which are crucial components of both natural and agricultural ecosystems. The contributions of selective and neutral processes in defining community composition are often confounded in field studies because as one moves through space, environments also change. Managed ecosystems provide an excellent opportunity to control for this and evaluate the relative strength of these processes by minimising differences between comparable niches separated at different geographic scales. We use next-generation sequencing to characterize the variance in fungal communities inhabiting adjacent fruit, soil and bark in comparable vineyards across $1000 \mathrm{kms}$ in New Zealand. By compartmentalizing community variation, we reveal that niche explains at least four times more community variance than geographic location. We go beyond merely demonstrating that different communities are found in both different niches and locations by quantifying the forces that define these patterns. Overall, selection unsurprisingly predominantly shapes these microbial communities, but we show the balance of neutral processes also have a significant role in defining community assemblage in eukaryotic microbes.
\end{abstract}

The ISME Journal (2015) 9, 2003-2011; doi:10.1038/ismej.2015.18; published online 10 March 2015

\section{Introduction}

Disentangling the processes regulating species' distributions makes a significant step towards achieving an integrated understanding of natural and managed ecosystems (Gaston, 2000). A better understanding of the factors underpinning community structure also ensures greater accuracy for predictions concerning both species conservation and communities' fates under various environmental change scenarios (Fierer et al., 2003; Lennon and Jones, 2011; Ferrenberg et al., 2013). Differences in the types and abundances of species in space may arise through both natural-selection-driven and non-selection driven processes, which are directly analogous to classic population genetic processes operating within species (Nemergut et al., 2013). Selective processes may drive differences between communities through species sorting in response to local conditions (Fierer and Jackson, 2006; Hughes et al., 2008); non-selective processes may generate variation through a combination of other assembly

Correspondence: P Morrison-Whittle, The School of Biological Sciences, The University of Auckland, Private Bag 92019, Auckland 1142, New Zealand.

E-mail: pmor072@aucklanduni.ac.nz

Received 2 July 2014; revised 9 December 2014; accepted 13 January 2015; published online 10 March 2015 processes including dispersal limitation, community drift and speciation (Fierer et al., 2003; Vellend, 2010; Hanson et al., 2012; Nemergut et al., 2013). These non-selective processes-which we define here as 'neutral' processes-are considered in ecological neutral theories (Bell, 2001; Hubbell, 2005) and are predicted to produce variation in community structure through space without needing to invoke the actions of selection.

While we have a limited understanding of processes that drive broad community patterns for macrobes (Gaston, 2000; Wiens, 2011), we have far less data on how these translate to microbial communities, which are crucial components of natural, agricultural and biotechnological ecosystems (Bardgett et al., 2008; Van Der Heijden et al., 2008; Fuhrman, 2009; Hanson et al., 2012). Due to their cryptic nature, it is relatively more challenging to examine patterns in microbial than macrobial communities. Massively parallel next-generation sequencing technologies have allowed significant leaps forward in the power with which we may sample microbial communities. These methods do not have the constraint of having to culture species to analyse them, but instead evaluate DNA directly isolated from the substrate of interest (Su et al., 2012; Kautz et al., 2013; Bokulich et al., 2014; Taylor et al., 2014). So far, studies utilising these techniques are providing increasing evidence that many microbial 
communities are not homogenised through space, but display significant structure just like many plant and animal communities do (Martiny et al., 2006; Hanson et al., 2012; Nemergut et al., 2013). These findings have principally been based on studies examining bacteria (Fierer and Jackson, 2006; Bryant et al., 2008; Fulthorpe et al., 2008; Lauber et al., 2009; Knief et al., 2010; Ghiglione et al., 2012); the relatively fewer studies examining eukaryotic microbes also show differential patterns of community composition in space (Dumbrell et al., 2010; Scheckenbach et al., 2010; Bokulich et al., 2014; Taylor et al., 2014).

Early speculations concerning the forces that regulate microbial community composition are represented by the classic Baas Becking hypothesis 'everything is everywhere but the environment selects'. This suggests that, due to small individual sizes but large populations, dispersal is not limiting and that only selection due to environmental gradients accounts for differences in community structure (De Wit and Bouvier, 2006). This idea directly informs a pervading view which implicitly assumes that the actions of natural selection dominate, and many microbial community ecology studies have correspondingly only attempted to evaluate the role of selection in community assemblage. Overall there has been less focus on testing whether microbial communities may become differentiated as a consequence of various neutral processes (Hanson et al., 2012; Nemergut et al., 2013). While it is unrealistic to imagine that only one or the other of these 'selective' or 'neutral' processes are at play, it is not clear how important each process is in influencing any structure we observe. Consequently, without accounting for relative contribution of neutral processes we cannot assume the dominance of selection without empirically testing this first. In some sense the field could be considered in an analogous position to evolutionary biology before Gould and Lewontin's classic 'Spandrels' paper (Gould and Lewontin, 1979) which suggested that organisms' traits must be proved to have arisen via natural selection rather than assume they have.

The scant data attempting to disentangle these two drivers of microbial community composition are conflicting, and primarily come from studies with bacteria (Hanson et al., 2012). Studies examining gastrointestinal and soil subsurface bacterial communities show the primacy of selection, but also indicate that neutral processes have a secondary role (Jeraldo et al., 2012; Stegen et al., 2012, 2013; Wang et al., 2013). In contrast bacterial communities in wastewater plants appear primarily defined by neutral community assemblage (Ofițeru et al., 2010), and neutral effects primarily influence desert photosynthetic bacterial assemblages (Caruso et al., 2011). Dumbrell et al. (2010) evaluated the processes regulating the formation of soil arbuscular mycorrhizal fungal communities sampled less than $20 \mathrm{~m}$ apart, and showed these communities are shaped primarily by selection, but there was also a contribution from underlying neutral processes. However, we are unaware of any study that has tested and quantified the relative role of selection in defining eukaryotic microbial communities over larger scales, where processes like dispersal limitation and community drift may become increasingly important.

The difficulty facing studies attempting to separate and quantify the effects of neutral and selective processes as regulators of community structures is that as distance increases, environmental similarity often decreases. Thus, studies that sample across reasonable distances tend also to sample different environments, confounding one's ability to determine whether neutral or selective processes largely define any observed differences in communities (Bell, 2001; Chase and Myers, 2011; Ranjard et al., 2013). So far statistical treatments have been developed to attempt to overcome this problem separating these effects (Dumbrell et al., 2010; Chase and Myers 2011; Stegen et al., 2012, 2013).

Here we employ a complimentary but alternative approach and experimentally evaluate community diversity in three adjacent niches, within artificially managed ecosystems (vineyards), replicated across a range of spatial scales.

Vineyards naturally harbour a range of microbial taxa including diverse fungal communities (Bokulich et al., 2014; Pinto et al., 2014; Taylor et al., 2014). The conserved design and management of vineyard ecosystems (which comprise the same clone of plant, with comparable associated habitats and niches) across large distances provide a powerful system to separately estimate the impact of selective and neutral processes. Within any one vineyard, there are no apparent barriers to prevent the mixing of species across physically adjacent niches through, for example, insect mediated dispersal (Buser et al., 2014). At the same time, these niches are separated ecologically by multiple environmental gradients that provide an opportunity for selection to influence community composition. Such habitat comparisons are rarely possible using unmanaged ecosystems, which vary more greatly in space, and frequently compound extreme barriers to dispersal and environmental gradients.

Here, we use next-generation sequencing to evaluate 106 contemporaneous fungal communities inhabiting adjacent soil, bark and fruit niches across six New Zealand regions spanning a thousand kilometres. Using this design, we empirically test and compare the effect of both selective and neutral processes on community structure using permutational multivariate ANOVA of community dissimilarities. If selection dominates then communities will tend to differ more greatly between niches regardless of distance, and if neutral processes dominate then communities will tend to differ more greatly by distance regardless of niche. Here, we attempt a major step forward by not just robustly 
estimating whether any microbial community patterns exist, but going on to experimentally quantify the relative contributions of selective and neutral processes in defining community composition (Chase and Myers, 2011; Hanson et al., 2012).

\section{Materials and methods}

\section{Sampling and extraction}

Approximately $30 \mathrm{~g}$ of vine bark, soil and ripe fruit were aseptically sampled from Vitis vinifera var. Sauvignon blanc vineyards across six regions of $\mathrm{New}$ Zealand: Hawkes Bay, Martinborough, Nelson, Central Otago and the Wairau and Awatere valleys in Marlborough. These regions span approximately $38^{\circ}-45^{\circ} \mathrm{S}$ and $168^{\circ}-177^{\circ} \mathrm{E}$, around $1000 \mathrm{~km} \mathrm{NE}$ to SW gradient (Figure 1b). Six vineyards were selected in each region, and three sub-samples were taken evenly across each vineyard for each niche and pooled for a total of 108 samples (three niches sampled across 36 vineyards). All samples were taken at least $5 \mathrm{~m}$ from row ends to avoid edge effects. Soil samples were taken from directly under vines $50 \mathrm{~cm}$ away from the central trunk. All samples were taken approximately 2 weeks before harvest in April 2011 and transported to the laboratory in sterile containers on ice. Fruit samples were washed with $300 \mathrm{ml}$ of sterile water to remove epiphytes and then centrifuged; the resulting pellet was re-suspended in $500 \mu \mathrm{l}$ of sterile water. Before DNA extraction, all samples were stored at $-20^{\circ} \mathrm{C}$. DNA was extracted from all samples using the Zymo Research Soil Microbe DNA MiniPrep kits (Irvine, CA, USA). We empirically determined this procedure was sufficient to extract DNA from fruit and bark samples as well as soil (data not shown). The 600-bp D1/D2 region of the $26 \mathrm{~S}$ ribosomal RNA locus was amplified using the NL1 and NL4 fungalspecific primers (Kurtzman and Robnett, 2003). This locus provides a readily aligned homogeneous PCR product and also provides good signal for community differentiation (Taylor et al., 2014). Thirty-six different multiplex identifiers were added to the primers to bioinformatically distinguish between samples. Two fruit samples failed to amplify from raw DNA extractions, reducing the total number of samples to 106. PCR products were cleaned with AmpureXP beads to remove all primer dimers, and their quality confirmed with Agilent DNA1000 chips (Santa Clara, CA, USA). PCR products were uni-directionally sequenced on a full plate of a 454 Life Sciences GS FLX instrument by Macrogen (Seoul, Korea).

\section{Data analysis}

Sequence handling and processing was conducted with Mothur v. 1.30 (Schloss et al., 2009). Raw sequences for each sample are present in GenBank (accession number: SRP048520). Initially primers and reads $<200 \mathrm{bp}$ were removed, and then lowquality reads and homopolymer errors were identified and removed using the pyronoise algorithm. PCR chimeras were identified and removed using the uchime algorithm. Individual sample identifiers were assigned to the resulting good quality reads, which were then merged and analysed together. Unique sequences were identified and compared with a fungal reference database, and those not assigned to Fungi $(6.76 \%$ of all unique sequences, $5.75 \%$ of reads) were removed. While there will likely be differential genetic diversity within fungal species, empirical studies suggest that multiple species of Ascomycota and Basidiomycota differ by less than $2 \%$ at the $26 \mathrm{~S}$ rDNA gene (Kurtzman and Robnett, 2003; Romanelli et al., 2010). Thus, the remaining 486279 reads were aligned against a fungal reference database and clustered into groups that share $>98 \%$ identity. These are considered as the lowest level of molecular operational taxonomic units (MOTUs) and approximate species. We conservatively removed any 'singleton' MOTUs, those represented by just a single read, from all further analyses. An unequal number of reads were obtained across samples (ranging from 1257 to 8007), and so we re-sampled (rarefied) each community down to
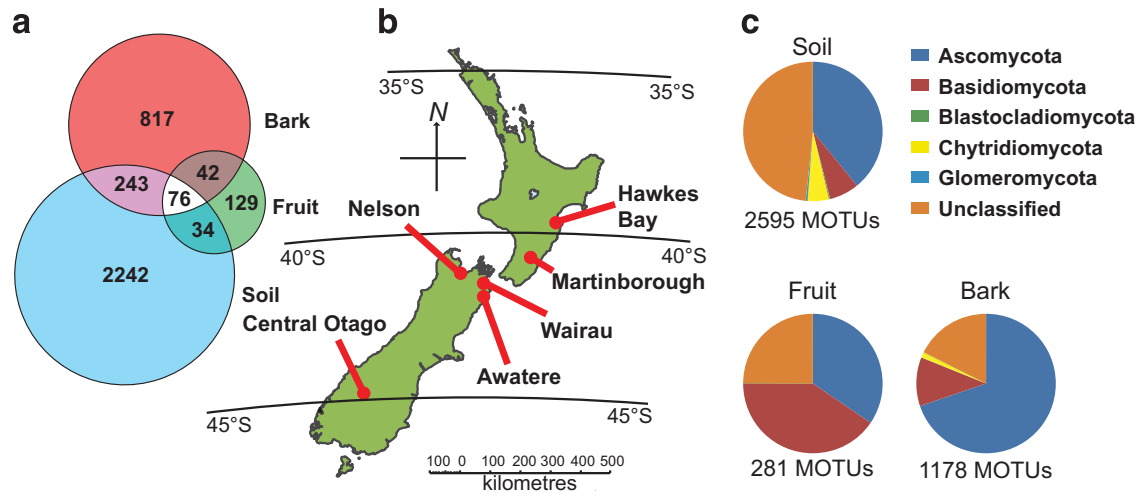

Figure 1 (a) Community diversity (number of species) among all three vineyard niches (bark, fruit and soil) and their overlap. (b) The six New Zealand wine-growing regions sampled. (c) Relative proportions of total MOTU diversity among niches for the five fungal phyla detected. 
the sample with the lowest number of reads to produce a data set with equivalent sampling effort for all communities, and this totalled 133454 reads. MOTUs were compared with a fungal taxonomic database using a Bayesian approach, and each was classified to all levels above genus with the 'classify. seqs' command in Mothur. Consensus sequences that matched less than $70 \%$ at any one taxonomic level were listed as unclassified; the final data set is provided in Supplementary Table S1.

\section{Statistical analysis}

Following Crist et al. (2003) we employed null models to test whether there is evidence to support the idea that any variance in observed species richness is no more than we would expect to see by chance given a random distribution of individuals across our samples. Species richness can be partitioned into $\alpha$-diversity (the average number of species per sample unit), $\beta$-diversity (species richness difference between the average sample unit and the overall species pool) and $\gamma$-diversity (the total number of species observed in a defined area). $\alpha$ - and $\beta$-diversity was analysed for all three niches across all vineyards independently and compared with $\alpha$ - and $\beta$-diversity measures predicted by randomisation simulations. Community null models are typically employed to analyse variation within niches and to our knowledge no analytical tools have been designed to allow inclusion of multiple niches. To verify whether within-niche regional patterns of richness held for overall vineyard richness, we additionally tested whether overall vineyard richness was randomly assembled by pooling species presence from each of the three vineyard niches. Our experimental design, sampling replicate vineyards in replicate regions, allows comparisons of average species richness between vineyards and both their regional and national species richness.

To more comprehensively analyse the effects of niche and geographic location on both community composition (species richness) and community structure (species abundances), we conducted a two-way permutational multivariate ANOVA (Permanova) using a Jaccard (metric Bray-Curtis) community dissimilarity metric (PERMANOVA; Anderson, 2001). These comparisons were then replicated using a number of other metrics
(Bray-Curtis, Euclidean and Manhattan). Permanova tests were also conducted separately with both niche and region as fixed effects, and the BenjaminiHochberg multiple test correction (Benjamini and Hochberg, 1995) was employed to evaluate the suite of results. Variance in community structure described by Jaccard dissimilarities was visualised using principal coordinate analysis in $\mathrm{R}$ ( $\mathrm{R}$ Development Core Team, 2013) and graphed using JMP (SAS Institute Inc, 1989-2007). Mantel tests testing for correlations between community dissimilarity and geographic spatial separation were conducted for each niche separately. In addition, since we have sequence data for all taxa, we evaluated communities using weighted-unifrac analysis (Lozupone et al., 2006). This analysis accounts for species abundances as well as phylogenetic relatedness and uses randomisation to assess the degree to which observed community phylogenetic dissimilarity differs from those expected randomly. All statistical testing was carried out using the vegan package (Dixon, 2003) in $\mathrm{R}$ with the exception of weighted-unifrac analysis that was performed in Mothur v. 1.30.

\section{Results}

\section{Overall fungal diversity}

Analyses of the sequences from the replicate soil, vine bark and fruit samples revealed the presence of 3583 fungal MOTUs (herein called species). Taxonomic assignment of representative sequences for each species, by comparison with a fungal reference database, reveals members from five fungal phyla, 24 classes, 59 orders, 104 families and 166 genera (see Supplementary Appendix S1). The Ascomycota dominated comprising $45.3 \%$ of all MOTUs, followed by the Basidiomycota with $9.6 \%$. Table 1 shows species richness (i.e., presence/absence) broken down by both niche and region. Species richness significantly differed by niche but not by region (two-way ANOVA, $F_{[2,89]}=662.471, P<0.0001$ and $\mathrm{F}_{[5,89]}=0.942, P=0.457$, respectively). In contrast to evidence for latitudinal patterns in animal communities (Gaston, 2000), there was no significant correlation between latitude and species richness for our sampled fungal communities in bark, fruit or soil (Bark: $P<0.117$ and $r<0.266$, Fruit: $P<0.095$

Table 1 Species richness by region and niche

\begin{tabular}{|c|c|c|c|c|c|c|c|}
\hline & Hawkes Bay & Martinborough & Nelson & Wairau & Awatere & Central Otago & All regions \\
\hline Bark & 402 & 411 & 411 & 394 & 297 & 334 & 1178 \\
\hline Fruit & 82 & 88 & 57 & 90 & 92 & 99 & 281 \\
\hline Soil & 830 & 849 & 808 & 858 & 752 & 845 & 2595 \\
\hline All niches & 1202 & 1214 & 1171 & 1217 & 1037 & 1131 & \\
\hline
\end{tabular}

The numbers of species in all regions and niches are not the sum of the rows and columns as many species are present in more than one niche and location. 
and $r<-0.291$, Soil: $P<0.954$ and $r<0.010)$. While the total number of species in each region was approximately similar (averaging around 1100), there were 9-fold and 4-fold more species in soil and bark than in fruit, respectively. Just $2.1 \%$ (76 species, Figure 1a) of species were found in all three adjacent niches and accounted for 55.1, 93.7 and $37.1 \%$ of all reads in bark, fruit and soil communities, respectively. If the number of reads assigned to each species is reasonably assumed to represent taxa abundance, then species abundance approximately follows the typical power law decay distribution seen in communities generally (Bell, 2001). A breakdown of the abundance of major phyla in all three niches is shown in Figure 1c, and the breakdown over all taxonomic levels is shown in Supplementary Appendix S1. Approximately 84, 72 and $87 \%$ of species are unclassified at the genus level in bark, fruit and soil, respectively, indicating the relative extent of uncharacterised fungal diversity in these niches. The diversity of fungal species relative to sampling effort reported here is approximately in line with the only two other studies that employed next-generation sequencing approaches to analyse fungal communities associated with fruit in vineyards: 253 MOTUs in Taylor et al. (2014) and 158 MOTUs in Bokulich et al. (2014). However, fungal diversity in these $36 \mathrm{NZ}$ vineyard soils appears much greater than estimates from just two Italian vineyards with around 300 MOTUs in Orgiazzi et al. (2012), but one might expect greater diversity with a greater sampling area. Previous reports of fungal diversity found on vine bark derived from live culture only and yielded just five species (Sabate et al., 2002); however, next-generation sequencing of fungal communities from vine leaves reveals around a thousand MOTUs and is thus in line with our inferences from bark here (Pinto et al., 2014).

\section{Testing for patterns of community differences}

Our aim was to quantify the relative roles of selective and neutral processes in defining differences in communities, but this aim critically requires that differences exist. We first tested whether any patterns in community composition were evident in these data. The results of our additive diversity partitioning hierarchical null model test revealed that both average vineyard and average regional diversity were significantly lower than expected under a random species distribution across these New Zealand sample sites $(P<0.0001$; see Supplementary Appendix S2). Consequently, both regional and national $\beta$-diversity were significantly different than predicted from random models $(P<0.0001$; see Supplementary Appendix S2). Average national $\beta$-diversity (11.1) is approximately three times greater than average regional $\beta$-diversity (3.6), which shows individual communities on average tend to be more similar to other communities within their region than to those of other regions. These patterns of $\alpha$ - and $\beta$-diversity hold for each niche separately and for overall vineyard diversity. Together, these results provide support to reject the concept that these communities are assembled randomly, at least in terms of species presence and absence.

Permanova analyses also accounting for species abundances revealed that both geographic location and niche significantly affect both community diversity and composition (see Table 2). The significance of these effects and their relative $R^{2}$ values were stable when this analysis was conducted using a range of different community dissimilarity measures (Bray-Curtis, Euclidean, Manhattan; see Supplementary Appendix S3). These effects can be visualised in the first two dimensions of classic multidimensional scaling of Jaccard dissimilarities, and this is shown in Figure 2. Reducing the data to include only those species found in all regions (i.e., region-specific species removed) did not greatly affect the $P$ or $R^{2}$ values $\left(P<0.0001, R^{2}=0.070\right)$, indicating that regional differentiation is not purely a product of region-specific species, but differences in the proportion of shared species. The results of permanova also revealed a significant interaction between both niche and region (see Table 2). To dissect this interaction, we explored the 63 possible comparisons of communities between niches within regions, and between regions for each niche. Once multiple-testing correction had been applied, these unsurprisingly revealed differential patterns of regional delineations for niches (see Supplementary Appendix S4), with soil communities displaying the greatest number of significant regional differences. The results of weighted-unifrac analysis also reveal a significant effect of both niche and location on community composition (average niche comparison $\mathrm{W}$-score $=1, P<0.001$; average regional comparison $\mathrm{W}$-score $=0.65, \quad P<0.001 ; \quad$ see Supplementary Appendix S5). Together, these various tests converge to reveal a significant effect of both niche and geographic location on fungal community composition.

\section{The effect of geographic distance on ecological dissimilarity}

Mantel correlations between Jaccard community dissimilarities and geographic distance matrices carried out for each niche independently revealed

Table 2 Results of permutation ANOVA of Jaccard dissimilarities between fungal communities found in New Zealand vineyards (9999 permutations)

\begin{tabular}{lrrrrrc}
\hline Effect & $d f$ & \multicolumn{1}{c}{$S S$} & $M S$ & Pseudo-F & $\mathrm{R}^{2}$ & $\mathrm{P}$ \\
\hline Region & 5 & 3.017 & 0.603 & 2.211 & 0.07 & $<0.001$ \\
Niche & 2 & 11.125 & 5.562 & 20.383 & 0.257 & $<0.001$ \\
Region $\times$ Niche & 10 & 5.144 & 0.514 & 1.885 & 0.119 & $<0.001$ \\
Residuals & 88 & 24.014 & 0.273 & 0.555 & & \\
\hline
\end{tabular}

Abbreviations: MS, mean square error; SS, sum of squares. 


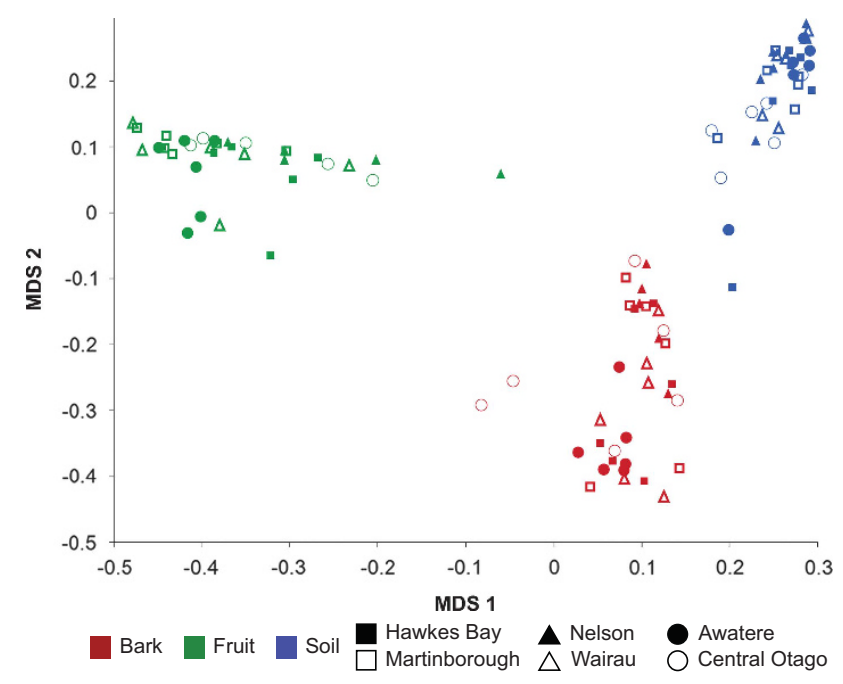

Figure 2 First two dimensions of classic multidimensional scaling of Jaccard distances between samples from different niches and regions.

no effect of distance on bark communities, a borderline-significant correlation for fruit, and a significant correlation for soil communities (bark: $P<0.111, r=0.103$; fruit: $P=0.050, r=0.148$; soil: $P<0.0001, \quad r=0.355)$. Within-region mantel tests examining correlations between community and geographic distance for both soil and fruit niches show this relationship breaks down at smaller distances. The average distance between sites within regions is $12 \mathrm{~km}$, and the maximum is around $100 \mathrm{~km}$ (see Supplementary Appendix S6). After multiple pairwise test correction, only one of the 18 within region niche-specific community mantel tests reported a significant relationship. This result suggests that $100 \mathrm{~km}$ is the cutoff for similarity of fungal communities inhabiting soil and possibly fruit niches.

\section{Quantifying the effects of selection and neutral processes}

Having demonstrated the significant effects of both niche and geographic location on fungal community composition, we went on to quantify the relative amount of observed variance in community diversity and composition explained by these factors. The $R^{2}$ values derived from permanova analyses allow us to assess this directly. Using community distance metrics based on only the presence/absence of species, geographic location explains an average of $6.1 \%$ of the variance in community diversity, but niche explains $27.1 \%$ (evaluated using both binary Bray-Curtis and Jaccard). When species abundances are taken into account, the average proportion of the total variation explained by niche was $31.9 \%$, approximately four times the proportion of variation explained by region at just $7.2 \%$. Thus, while both location and niche, and their interaction, appear to have a significant role in defining differences in eukaryotic microbial community diversity and composition, niche of isolation appears to explain at least four times more of the community variance than geographic location. Given this experimental design and analysis this shows that both selective and neutral effects have a role in defining fungal communities, but that selective effects are approximately four times stronger.

\section{Discussion}

We sampled fungal communities from three different adjacent niches that are relatively conserved at scales of metres to thousands of kilometres with the aim of quantifying the relative degree to which selective and neutral processes define community composition. Direct sequencing of DNA from these niches reveals approximately the same extent of fungal diversity recovered from similar niches in the handful of other studies that have used this approach (Bokulich et al., 2014; Pinto et al., 2014; Taylor et al., 2014). These communities are not homogenous; they significantly vary in terms of species richness and community composition, and both niche and geographic location affect this variance. In addition, there is a significant interaction between these main effects, and this is likely driven by the fact that while communities in each niche demonstrate geographic differences overall, the nature of these geographic patterns is slightly different within each of these three niches (see Supplementary Appendix S4). While the extent of latitude covered is relatively small, these data show no significant correlation between species richness and latitude for communities in any niche. This finding aligns with the lack of evidence for latitudinal patterns reported for microbes (Fierer and Jackson, 2006), and contrasts diversity patterns of macro-organisms (Gaston, 2000; Hillebrand, 2004). Further, we provide evidence that there is significant distance decay between fungal communities, within at least soils, over distances of about $100 \mathrm{~km}$. This overall result nicely recapitulates patterns revealed from the population genetic analyses of just one species in this community sourced from these same samples: Saccharomyces cerevisiae (Knight and Goddard, 2014). The recovery of the same patterns at both the species and community level lends confidence to the ability of these data to reveal underlying biological signals.

By estimating the relative strength of selective and neutral processes in generating variation in community structure, these results go beyond merely demonstrating that different species are found in different places (either by niche or by geography). Given the individually conserved adjacent niches sampled over varying distances, these data show that communities tend to be more similar by niche than by distance. Selective forces will drive similarities between niches regardless of distance, and a range of neutral forces will drive similarities between geographic locations regardless of niche. Crucially, 
this means that niche effects may explain approximately one-quarter of the variance in community composition, but only one-twentieth of the variance, four-fold less, may be explained by geographic location. While regionally structured communities have been observed in vineyard communities before (Gayevskiy and Goddard, 2011; Bokulich et al., 2014; Taylor et al., 2014), this is the first study to estimate the relative strength of selective and neutral processes in defining microbial communities across regional scales for this managed ecosystem. Currently, there are no studies examining whether this pattern holds more broadly across other ecosystems, or at larger scales. Thus, these data start to fill the gap in our understanding of the relative magnitude of the forces that regulate community composition for eukaryotic microbes, which are crucial components of both natural and agricultural ecosystems. While our data show selection is influencing community structure to a greater degree than the neutral processes are, we show the balance of neutral forces is significant. In principle any of the neutral forces of differential speciation, community drift, dispersal limitation or their interaction might underlie the neutral component defining community difference in this system. Our experiment was not designed to tease these apart, and our data do not allow us to do this. Instead here we evaluate the net effect of these processes (within niche variation) compared with selective processes (between niche variation).

Our experimental design aimed to capture and control for variation in community structure in response to two separate and frequently co-varying and potentially confounding niche and distance effects. In our attempt to disentangle these effects, we make an assumption that communities residing in the same niche across different locations were exposed to approximately equivalent selection pressures. While vineyard ecosystems are managed and conserved by human design, these are ultimately open ecosystems. It is therefore unlikely that our assumption of ecological equivalence of the various niches across distances was fully satisfied. Of the three niches, soil likely differs most across NZ (Ranjard et al., 2013). Soil chemistry and physical properties were not controlled for in our sampling design, all of which have been implicated in microbial turnover (Dumbrell et al., 2010; Ranjard et al., 2013). As a consequence, it is reasonably likely that some of the differences between soil communities in different locations originate as a product of selection, not neutral effects. It seems reasonable that this effect will be lesser for the communities inhabiting bark and fruit as all samples originated from the same clonal variety of plant species. However, selective effects are unlikely to be entirely absent as different soils will likely translate into different plant phenotypes. We also acknowledge that region-specific variation may also have clearly arisen in all niches from climatic differences between regions. A number of environmental gradients exist across New Zealand's regions, such as temperature (although this varies just 2-3 degrees), precipitation rates and UV radiance. However, we feel these considerations do not prevent us from reaching meaningful inferences about the relative strength of the forces regulating these communities overall: if intra-niche community variation is jointly influenced by neutral processes and natural selection to undetected environmental variance, then intra-niche variation will simply represent the maximum limit of the influence of neutral processes. This means we can refine our summary by concluding that the influence of selection is at least four times stronger than neutral forces.

Very little work has been conducted to examine the relative balance of selection and neutral effects on regulating microbial community structure, and this has been largely focused on bacterial communities. However, our findings are in line with previous work by Stegen et al. (2012; 2013), who showed selection is the dominant effect shaping subsurface soil bacterial communities over small distances $(<53 \mathrm{~m})$ while neutral forces also have a role; and Bell (2010) who concluded that dispersal limitation had a minor role over distances of under $1 \mathrm{~km}$. Our findings are nicely in line with Dumbrell et al. (2010) who examined arbuscular mycorrhizal fungal communities less than $25 \mathrm{~m}$ apart, and concluded that niche partitioning was the primary mechanism regulating the composition and diversity of communities, but these communities are also influenced by neutral processes. Our results are also consistent with studies of larger plants and animals that indicate the influence of both selection and neutral effects on community composition (Turnbull et al., 2005; Farnon Ellwood et al., 2009). Broadly speaking then, along with other work, our results suggest the primacy of selection as the consistently strongest effect on community regulation over large scales, but show that balance of neutral effects is also important. How this balance changes across different ecosystems for different microbial taxa remains to be seen. While we know that neutral processes can generate variation, we do not know $a$ priori how great this variation is in any ecosystem, and must be measured, not assumed.

The primacy of selection in shaping communities generally gives rise to the prediction that environmental change will have a greater effect on community composition than if neutral forces were the dominant driver of community structure. Humanmediated habitat change and irregular weather events with greater extremes of conditions predicted under climate change models will likely manipulate the nature of selective forces and thus affect macrobial and microbial communities in both natural and managed agricultural ecosystems. However, the absolute speed with which species within these communities adapt to these changes will likely be greater for microbes due to their faster generation times and larger population sizes (Goddard and Bradford, 2003). 


\section{Conflict of Interest}

The authors declare no conflict of interest.

\section{Acknowledgements}

We thank Sarah Knight and Soon Lee who assisted in sample collection and processing, Peter Tsai for bioinformatic assistance and Alexandria Leonard for her assistance in editing the manuscript. This work was funded by grants to MG from the New Zealand Ministry of Business, Innovation and Employment, Plant and Food Research Ltd and New Zealand Winegrowers. The completion of this research would not have been possible without the cooperation and assistance of the many collaborating companies who allowed access to their land: Amisfeild, Ata Rangi, Churton, Coal Pit, Constellation, Delegats, Domain Road, Frey Vineyard, Huia, Misha's Vineyard, Mt Difficulty, Mt Riley, Neudorf, Palliser, Pernod Ricard, Rippon, Seifried, Seresin, Te Kairanga, Te Whare Ra, Tohu, Trinity Hill, Villa Maria and Vita Brevis.

\section{References}

Anderson MJ. (2001). A new method for non-parametric multivariate analysis of variance. Austr Ecol 26: 32-46.

Bardgett RD, Freeman C, Ostle NJ. (2008). Microbial contributions to climate change through carbon cycle feedbacks. ISME J 2: 805-814.

Bell G. (2001). Neutral macroecology. Science 293: 2413-2418.

Bell T. (2010). Experimental tests of the bacterial distancedecay relationship. ISME J 4: 1357-1365.

Benjamini Y, Hochberg Y. (1995). Controlling the false discovery rate: a practical and powerful approach to multiple testing. J $R$ Stat Soc Series B Methodol 57: 289-300.

Bokulich NA, Thorngate JH, Richardson PM, Mills DA. (2014). Microbial biogeography of wine grapes is conditioned by cultivar, vintage, and climate. Proc Natl Acad Sci USA 111: E139-E148.

Bryant JA, Lamanna C, Morlon H, Kerkhoff AJ, Enquist BJ, Green JL. (2008). Microbes on mountainsides: Contrasting elevational patterns of bacterial and plant diversity. Proc Natl Acad Sci USA 105: 11505-11511.

Buser CC, Newcomb RD, Gaskett AC, Goddard MR. (2014). Niche construction initiates the evolution of mutualistic interactions. Ecol Lett 17: 1257-1264.

Caruso T, Chan Y, Lacap DC, Lau MCY, McKay CP, Pointing SB. (2011). Stochastic and deterministic processes interact in the assembly of desert microbial communities on a global scale. ISME J 5: 1406-1413.

Chase JM, Myers JA. (2011). Disentangling the importance of ecological niches from stochastic processes across scales. Philos Trans $R$ Soc B Biol Sci 366: 2351-2363.

Crist TO, Veech JA, Gering JC, Summerville KS. (2003). Partitioning species diversity across landscapes and regions: a hierarchical analysis of $\alpha, \beta$, and $\gamma$ diversity. Am Nat 162: 734-743.

De Wit R, Bouvier T. (2006). 'Everything is everywhere, but, the environment selects'; what did Baas Becking and Beijerinck really say?Environ Microbiol 8: 755-758.
Dixon P. (2003). VEGAN, a package of $\mathrm{R}$ functions for community ecology. J Veg Sci 14: 927-930.

Dumbrell AJ, Nelson M, Helgason T, Dytham C, Fitter AH. (2010). Relative roles of niche and neutral processes in structuring a soil microbial community. ISME $J$ 4: 337-345.

Farnon Ellwood MD, Manica A, Foster WA. (2009). Stochastic and deterministic processes jointly structure tropical arthropod communities. Ecol Lett 12: 277-284.

Ferrenberg S, O'Neill SP, Knelman JE, Todd B, Duggan S, Bradley D et al. (2013). Changes in assembly processes in soil bacterial communities following a wildfire disturbance. ISME J 7: 1102-1111.

Fierer N, Jackson RB. (2006). The diversity and biogeography of soil bacterial communities. Proc Natl Acad Sci USA 103: 626-631.

Fierer N, Schimel JP, Holden PA. (2003). Influence of drying-rewetting frequency on soil bacterial community structure. Microb Ecol 45: 63-71.

Fuhrman JA. (2009). Microbial community structure and its functional implications. Nature 459: 193-199.

Fulthorpe RR, Roesch LFW, Riva A, Triplett EW. (2008). Distantly sampled soils carry few species in common. ISME J 2: 901-910.

Gaston KJ. (2000). Global patterns in biodiversity. Nature 405: 220-227.

Gayevskiy V, Goddard MR. (2011). Geographic delineations of yeast communities and populations associated with vines and wines in New Zealand. ISME J 6: 1281-1290.

Ghiglione JF, Galand PE, Pommier T, Pedrós-Alió C, Maas EW, Bakker K et al. (2012). Pole-to-pole biogeography of surface and deep marine bacterial communities. Proc Natl Acad Sci USA 109: 17633-17638.

Goddard MR, Bradford MA. (2003). The adaptive response of a natural microbial population to carbon- and nitrogen-limitation. Ecol Lett 6: 594-598.

Gould SJ, Lewontin RC. (1979). The spandrels of San Marco and the Panglossian paradigm: a critique of the adaptationist programme. Proc $R$ Soc Lond B Biol Sci 205: 581-598.

Hanson CA, Fuhrman JA, Horner-Devine MC, Martiny JBH. (2012). Beyond biogeographic patterns: processes shaping the microbial landscape. Nat Rev Microbiol 10: 497-506.

Hillebrand H. (2004). On the generality of the latitudinal diversity gradient. Am Nat 163: 192-211.

Hubbell SP. (2005). Neutral theory in community ecology and the hypothesis of functional equivalence. Funct Ecol 19: 166-172.

Hughes AR, Inouye BD, Johnson MTJ, Underwood N, Vellend M. (2008). Ecological consequences of genetic diversity. Ecol Lett 11: 609-623.

Jeraldo P, Sipos M, Chia N, Brulc JM, Dhillon AS, Konkel ME et al. (2012). Quantification of the relative roles of niche and neutral processes in structuring gastrointestinal microbiomes. Proc Natl Acad Sci USA 109: 9692-9698.

Kautz S, Rubin BER, Russell JA, Moreaua CS. (2013). Surveying the microbiome of ants: Comparing 454 pyrosequencing with traditional methods to uncover bacterial diversity. Appl Environ Microbiol 79: 525-534.

Knief C, Ramette A, Frances L, Alonso-Blanco C, Vorholt JA. (2010). Site and plant species are important determinants of the Methylobacterium community 
composition in the plant phyllosphere. ISME $J$ 4: 719-728.

Knight S, Goddard MR. (2014). Quantifying separation and similarity in a Saccharomyces cerevisiae metapopulation. ISME J 9: 361-370.

Kurtzman CP, Robnett CJ. (2003). Phylogenetic relationships among yeasts of the 'Saccharomyces complex' determined from multigene sequence analyses. FEMS Yeast Res 3: 417-432.

Lauber CL, Hamady M, Knight R, Fierer N. (2009). Pyrosequencing-based assessment of soil $\mathrm{pH}$ as a predictor of soil bacterial community structure at the continental scale. Appl Environ Microbiol 75: 5111-5120.

Lennon JT, Jones SE. (2011). Microbial seed banks: The ecological and evolutionary implications of dormancy. Nat Rev Microbiol 9: 119-130.

Lozupone C, Hamady M, Knight R. UniFrac - An online tool for comparing microbial community diversity in a phylogenetic context. BMC Bioinformatics 2006; 7: 371.

Martiny JBH, Bohannan BJM, Brown JH, Colwell RK, Fuhrman JA, Green JL et al. (2006). Microbial biogeography: Putting microorganisms on the map. Nat Rev Microbiol 4: 102-112.

Nemergut DR, Schmidt SK, Fukami T, O'Neill SP, Bilinski TM, Stanish LF et al. (2013). Patterns and processes of microbial community assembly. Microbiol Mol Biol Rev 77: 342-356.

Ofițeru ID, Lunn M, Curtis TP, Wells GF, Criddle CS, Francis CA et al. (2010). Combined niche and neutral effects in a microbial wastewater treatment community. Proc Natl Acad Sci USA 107: 15345-15350.

Orgiazzi A, Lumini E, Nilsson RH, Girlanda M, Vizzini A, Bonfante $\mathrm{P}$ et al. (2012). Unravelling soil fungal communities from different mediterranean land-use backgrounds. PLOS ONE 7: e34847.

Pinto C, Pinho D, Sousa S, Pinheiro M, Egas C, Gomes AC. (2014). Unravelling the diversity of grapevine microbiome. PLoS ONE 9: e85622.

R Development Core Team (2013). R: A Language and Environment for Statistical Computing. R Foundation for Statistical Computing: Vienna, Austria.

Ranjard L, Dequiedt S, Chemidlin Prévost-Bouré N, Thioulouse J, Saby NPA, Lelievre M et al. (2013). Turnover of soil bacterial diversity driven by widescale environmental heterogeneity. Nat Commun 4: 1434.

Romanelli AM, Sutton DA, Thompson EH, Rinaldi MG, Wickes BL. (2010). Sequence-based identification of filamentous basidiomycetous fungi from clinical specimens: a cautionary note. J Clin Microbiol 48: 741-752.

Sabate J, Cano J, Esteve-Zarzoso B, Guillamón JM. (2002). Isolation and identification of yeasts associated with vineyard and winery by RFLP analysis of ribosomal genes and mitochondrial DNA. Microbiol Res 157: 267-274.

SAS Institute Inc. $J M P^{\circledR}$. SAS Institute Inc.: Cary, NC, 1989-2007.

Scheckenbach F, Hausmann K, Wylezich C, Weitere M, Arndt H. (2010). Large-scale patterns in biodiversity of microbial eukaryotes from the abyssal sea floor. Proc Natl Acad Sci USA 107: 115-120.

Schloss PD, Westcott SL, Ryabin T, Hall JR, Hartmann M, Hollister EB et al. (2009). Introducing mothur: Opensource, platform-independent, community-supported software for describing and comparing microbial communities. Appl Environ Microbiol 75: 7537-7541.

Stegen JC, Lin X, Fredrickson JK, Chen X, Kennedy DW, Murray CJ et al. (2013). Quantifying community assembly processes and identifying features that impose them. ISME J 7: 2069-2079.

Stegen JC, Lin X, Konopka AE, Fredrickson JK. (2012). Stochastic and deterministic assembly processes in subsurface microbial communities. ISME $J \quad \mathbf{6}$ : 1653-1664.

Su C, Lei L, Duan Y, Zhang KQ, Yang J. (2012). Cultureindependent methods for studying environmental microorganisms: Methods, application, and perspective. Appl Microbiol Biotechnol 93: 993-1003.

Taylor MW, Tsai P, Anfang N, Ross HA, Goddard MR. (2014). Pyrosequencing reveals regional differences in fruit-associated fungal communities. Environ Microbiol 16: $2848-2858$.

Turnbull LA, Manley L, Rees M. (2005). Niches, rather than neutrality, structure a grassland pioneer guild. Proc R Soc B Biol Sci 272: 1357-1364.

Van Der Heijden MGA, Bardgett RD, Van Straalen NM. (2008). The unseen majority: soil microbes as drivers of plant diversity and productivity in terrestrial ecosystems. Ecol Lett 11: 296-310.

Vellend M. (2010). Conceptual synthesis in community ecology. Q Rev Biol 85: 183-206.

Wang J, Shen J, Wu Y, Tu C, Soininen J, Stegen JC et al. (2013). Phylogenetic beta diversity in bacterial assemblages across ecosystems: Deterministic versus stochastic processes. ISME J 7: 1310-1321.

Wiens JJ. (2011). The niche, biogeography and species interactions. Philos Trans $R$ Soc B Biol Sci 366: 2336-2350.

Supplementary Information accompanies this paper on The ISME Journal website (http://www.nature.com/ismej) 